\title{
Research on Key Technology of Fault Diagnosis of Power Cables
}

\author{
Dandan Su ${ }^{1, a}$, Dezhong Kong ${ }^{1, b}$ \\ ${ }^{1}$ Wuxi Institute of Arts \& Technology, Wuxi, Jiangsu, China, 214206 \\ ${ }^{\mathrm{a}}$ email, ${ }^{\mathrm{b}}$ email
}

Keywords: Power Cables, Fault Diagnosis, Neural Network

\begin{abstract}
With the expansion of city scale and the acceleration of the process of urban construction, the power cables plays more and more important role in the city network. At the same time, the increase of the number of cables and the continuous extension of the cable running time result in more and more frequent cable failures. This paper analyzes the common causes of the cable fault, and gives the key technology of the cable fault diagnosis to provide some references for the relative researchers.
\end{abstract}

\section{Introduction}

With China's sustained and rapid economic development, accelerating the pace of city construction, the traditional erected over the city distribution overhead line and power supply equipment, equipped with the column, has been unable to meet the requirements of the development of city construction. Therefore, in the process of building urban distribution network in China, the main power supply products have been mainly used in underground power cables. Early cable fault caused by insulation aging, is easy to produce partial discharge; after the external force and chemical substances such as a series of effects, resulting in insulation fault cannot be recovered. With the cable running time, the insulation resistance of the cable began to slow down. There will be security risks. When the insulation is damaged, the partial discharge phenomenon is required to replace the cable in time, if it will lead to the continuous decline of the insulation resistance of the cable, and then develop into a permanent cable fault. A prerequisite to ensure safe and reliable operation of the power cable to the power cable insulation testing, in the past, want to overhaul the power cable fault, to power can be carried out, that is an offline detection technology, more time-consuming, but also caused by power loss is very great. In recent years, due to the increasing of the power supply, the problems of traditional methods exposed has been unable to adapt to the development of the electric power system, to realize the maintenance and repair of power cable online detection technology using power cable, through the use of a feature signal effectively find fault analysis method of power cable insulation, judge whether the occurrence of insulation breakdown, and then take appropriate emergency measures, to prevent and reduce the losses caused by the fault. The research on the fault analysis and diagnosis technology of power cable will be of great significance to the safe operation of power system, and to prolong the service life of the cable.

\section{Reasons for Cable Fault}

During the normal operation of power network cable, the insulation and protection layer are often affected by various factors. Such as the common vibration of crystallization fatigue, will lead to lead to the cable layer of lead to crack, and then affected by the environment affected by moisture, the impact on the insulation performance of the cable. Existence of a variety of cable running environment, is part of the cable in electric field environment, coupled with its internal containing 
air gap, the line insulation will be free inside the cable, to some extent also affects the performance of cable insulation. Cable during normal operation, if the situation appears in the ionization insulating medium, will appear in the air gap of the students into the ozone nitrate, so if you do not take timely measures to deal with, is bound to cause the corrosion of the cable insulation, the insulation properties decrease. Mechanical damage is one of the main points of cable fault processing; even smaller damage did not appear at the time of failure, after a period of time will continue to develop into a failure. Most of these faults are generated from the external force. Traffic overweight, digging and carrying etc. may cause cable damage, especially the vehicle vibration or impact load of the cable lead tape was damaged. In fact, the installation process is also the case of damage occurred. Operating behavior is not standardized, the installation of cable damage, or mechanical pull too large strain cable. In addition, the running environment of the power cable special, there will be deformation in the process of operation, it will appear on skin injury problems in the cable pipe or the support position, affect the safety of cable operation. Material is the key factor that determines the performance and quality of the power cable. With the rapid development of the cable manufacturing industry, more and more new materials are applied to the cable production. But because of the lack of practical production data, is very easy to cause the cable there are some quality defects in the manufacturing process, the problems such as wrinkles and cracks, damage the insulation wrapping process, the cable is put into use there is a big chance of failure.

\section{Key Technology of Fault Diagnosis of Power Cables}

Fault Tree Analysis. Fault Tree Analysis (short for FTA) is a system for special events do not want failure analysis interpretation, fault tree with a tree diagram representation of the logical relationship between the accident may occur and lead to accidents of all sorts of reasons, through the fault tree qualitative and quantitative analysis of the possible reasons for failure in order to prevent the accident, to provide a basis to improve the reliability of system. In order to know more about all the hidden troubles of the cable fault, the fault tree is used to make a qualitative analysis. Fault tree construction is the most important and the most basic link in FTA. The primary purpose of the contribution is to find the weak links in the system, and to take certain measures to improve the reliability of the system. In general, a predefined system does not want the event to occur as a top event. Next to find out all the results of the top event can be a fault event as an intermediate event of the fault tree. And then from the middle of the event to find out the cause of the occurrence of the event or until it can no longer be down analysis of the defects as the end of the event. At last, according to certain logic relation and specific sign, the fault tree diagram of the top event, the middle event and the bottom event of the fault tree is drawn. The purpose of qualitative analysis of the fault tree is to find out the possibility of the occurrence of the top event, so as to judge the weak link of the system and take appropriate measures to improve it. The task is to find out the minimum cut set of the fault tree, which is the direct cause of the failure of the top event. Fault tree through layers of in-depth analysis of the specific accident, which is convenient for the system to find the weak links to improve the security of the system; fault tree analysis has great flexibility. In addition to the analysis of the impact of a failure on the system, but also on the environmental impact caused by accidents and human factors, such as special reasons to be analyzed. We use the fault tree model not only to carry on the qualitative analysis, but also can carry on the quantitative analysis, in order to improve the system reliability and improve the reliability of the system.

Failure Mode Effect and Criticality Analysis. FMECA (Failure Mode Effect and Criticality Analysis) is a qualitative analysis method for reliability design. Its basic principle is according to 
the quality requirements, understand the system structure and operation environment, find the potential weak links analysis of the causes and effects of each failure mode may occur, each harm and influence to the system security, task success, maintenance and security resources etc. the improvement plan and to formulate relevant measures according to the degree of harm or compensation. The aim is to prevent and control the faults, to eliminate or reduce the defects in the design to improve the reliability of the system. FMECA is mainly composed of two parts: failure mode and effect analysis and hazard analysis. Among them, it is the analysis system of each potential failure mode and its causes and the system may bring the impact, as well as each fault mode according to the degree of harm to be classified. It mainly from the perspective of the risk of the complement and expansion, both qualitative and quantitative analysis, due to the system failure rate data cannot be determined, where the main qualitative analysis. According to the main points of FMECA, the general need basic information are identified, including object or unit name, fault mode, fault reason, fault effect, severity, detection method, compensation measures and evaluation with the form of description and concise list of all possible failure modes. We select the relevant fields to elect a representative individual, working together with the FMECA team. Through the collective wisdom, we can achieve mutual inspiration and information sharing, realizing quick cable diagnosis.

Wavelet Analysis. Based on the traditional Fourier transform signal analysis and processing, can guarantee the accuracy of the traditional parameters like current, voltage and power in the time domain, but can realize the measurement of the single frequency parameters in the frequency domain, but also reduce the time resolution, it is difficult to timely response to the influence factors of transient interference and waveform distortion of the system the method itself brings, is not also have the time domain and frequency domain analysis ability. In order to improve the accuracy of the fault signal detection in the power supply system, a new time-frequency analysis method based on wavelet analysis is adopted. This method not only has the adaptive ability of the signal, but also can change the time window and the frequency window, solves the problem of time and frequency resolution. The signal analysis and fault detection is an essential tool. Although the existing fault location method and fault locator can realize the fault location of power cable. However, due to the complexity of the electromagnetic environment of the power cable, there are some problems in the positioning accuracy and the processing of the fault signal. The wavelet analysis method can be used to decompose the current and voltage signals with singularity and instantaneous current, which can obviously reflect the fault signal at different scales. Based on the constructed distance function, the fault location of the fault signal is derived, and the fault location is finally reflected in the fault location. Compared with the traditional direct location method, the method of wavelet analysis can significantly improve the accuracy of fault location. Firstly, wavelet decomposition is used to describe the original signal of the power cable and then remove some of the components which are not needed.

Neural Network. Artificial Neural Network (short for Neural Network) is a complex dynamic network system, which is composed of a large number of neurons. It uses its own characteristics and rules to learn and identify and deal with complex problems within the system. The structure function of neural network is similar to the human brain, which can adapt to the environment. It has been widely used in the field of fault diagnosis and location, algorithm optimization, prediction and intelligent control. The advantages are as follows: the adaptive capability is strong; the distributed structure is beneficial to the processing of information; the execution speed is fast. Layered structure of neural network is adopted in most cases, according to whether the feedback between the layers can be divided into feed forward network and anti the network. Among them, the network is 
more typical of the back propagation network and radical basis function network, the main form of feedback network Hopfield network and recurrent neural network. back propagation network uses the algorithm has slow convergence speed and prediction accuracy is not obvious and easy to generate multiple local minima and a series of defects, neural network mainly used here is the radical basis function network, also called radial basis function network. The network is better than back propagation network in terms of convergence rate, recognition ability and prediction accuracy. Radical basis function network is a kind of forward neural network, is formed by a radial basis function neural network hidden layer and a linear output layer neurons. The radial basis function neural network between the values and weights specified in the range of radical basis function, improve the local approximation accuracy, so it is also called for local perception network. In order to achieve the purpose of cable fault diagnosis, using the method of combining wavelet analysis and neural network to realize neural network. According to the inherent characteristics of fast learning speed, high diagnosis accuracy, play a very important role in the diagnosis process. The first two steps of cable fault diagnosis are mainly obtained by simulation, and the conversion tool is used for format conversion, which is convenient for data preprocessing. Then the wavelet packet analysis is used to extract the feature signals, which has a direct impact on the fault diagnosis. The last is the construction of the neural network, mainly the establishment of two networks here were sub network fault identification and fault location network, through the pretreatment of the training samples and test samples to effectively implement the cable fault diagnosis.

\section{Conclusion}

Due to the special operation environment of power cable, the security and stability of power grid is often influenced by the various factors and quality problems. It is needed to choose the suitable detection method to find the fault location quickly and accurately. We should continue to accumulate experience to save manpower and material resources, shorten the time to deal with cable accidents and create greater economic and social benefits.

\section{References}

[1] Zhai Liang, Wen Yukun, Science and Technology \& Innovation, Vol. 2 (2015) No 22, p.114-115

[2] Tian Yu, Technology and Market, Vol. 21 (2014) No 12, p.45-47

[3] Liu Mengfei, Sui Xin, Low Carbon World, Vol. 6 (2015) No 8, p.34-35

[4] Ma Liang, Mechanical Engineer, Vol. 47 (2015) No 5, p.65-66 